\title{
Insuficiência Adrenal Relativa no Choque Séptico. Comparação da Resposta ao Tratamento com Hidrocortisona em Pacientes Diagnosticados através de Dosagem Única do Cortisol Plasmático Versus Teste da Corticotropina*
}

\author{
Relative Adrenal Insufficiency in Septic Shock. Comparison of the Response \\ to Hydrocortisone In Patients Diagnosed through Random Cortisol \\ Measurement Versus Corticotropin Test
}

José Raimundo A. de Azevedo', Renato Palácio de Azevedo², Rachel Jorge D. Cossetti ${ }^{3}$, Eduardo Rodrigues M. Lima ${ }^{3}$, Gescirene Borges de Sousa ${ }^{4}$

\section{RESUMO}

JUSTIFICATIVA E OBJETIVOS: A incidência de insuficiência adrenal relativa (IAR) no paciente com choque séptico é elevada e tem impacto significativo na sobrevida. O objetivo deste estudo foi determinar se a dosagem única do cortisol $<25 \mu \mathrm{g} / \mathrm{dL}$ é tão eficiente quanto o teste da corticotropina com $1 \mu \mathrm{g}$ para o diagnóstico de IAR em pacientes com choque séptico, avaliando a resposta hemodinâmica à administração de hidrocortisona.

MÉTODO: Os pacientes foram aleatorizados para

1. Diretor Executivo da Associação de Medicina Intensiva Brasileira (AMIB). Ex-Presidente da Sociedade Brasileira de Nutrição Parenteral e Enteral.

2. Estudante de Graduação. Faculdade de Medicina. Universidade Federal do Maranhão.

3. Médico Estagiário. UTI do Hospital São Domingos e UTI do Hospital Dr. Clementino Moura, São Luis, MA

4. Enfermeira da UTI do Hospital São Domingos. São Luis, MA.

${ }^{*}$ Recebido das Unidades de Terapia Intensiva do Hospital São Domingos e Hospital Dr. Clementino Moura. São Luis, MA.

Apresentado em 26 de dezembro de 2007

Aceito para publicação em 01 de fevereiro de 2008

Endereço para correspondência:

Dr. José Raimundo A. de Azevedo.

Diretor. UTI do Hospital São Domingos e UTI Hospital Dr. Clementino Moura.

Rua Osires Lote 6, Quadra 19, Apto 1201 - Renascença II

65075-775. São Luis, MA

Fax: (98) 3227-6798.

E-mail: jrazevedo@elo.com.br

(C)Associação de Medicina Intensiva Brasileira, 2008 dosagem única do cortisol ou teste da corticotropina com $1 \mu \mathrm{g}$. Após a coleta de sangue para dosagem do cortisol, os pacientes passaram a receber $100 \mathrm{mg}$ de hidrocortisona, por via venosa a cada 8 horas, durante 36 horas. IAR foi definida por uma dosagem única do cortisol $<25 \mu \mathrm{g} / \mathrm{dL}$ ou um $\Delta$ do cortisol $\leq 9 \mu \mathrm{g} / \mathrm{dL}$, após o teste da corticotropina.

RESULTADOS: Sessenta pacientes (G1 = 30; G2 = 30) foram incluídos na análise e mostraram-se comparáveis com relação aos dados demográficos, nosologias e gravidade. O tempo de uso de noradrenalina entre os pacientes do grupo $1 \mathrm{com}$ critério diagnóstico para IAR não diferiu daquele dos pacientes com cortisol $\geq 25$ $\mu \mathrm{g} / \mathrm{dL}$. No grupo 2, os pacientes com $\Delta$ cortisol $\leq 9 \mu \mathrm{g} /$ dL tiveram tempo de infusão de noradrenalina menor (3 dias) comparado aos pacientes com $\Delta$ cortisol $>9$ $\mu \mathrm{g} / \mathrm{dL}$ (6 dias).

CONCLUSÕES: O teste da corticotropina com $1 \mu \mathrm{g}$ foi mais eficiente que a dosagem única do cortisol plasmático $<25 \mu \mathrm{g} / \mathrm{dL}$, para o diagnóstico da insuficiência adrenal relativa no paciente com choque séptico.

Unitermos: Choque séptico, diagnóstico, Insuficiência adrenal relativa

\section{SUMMARY}

BACKGROUND AND OBJECTIVES: The incidence of relative adrenal insufficiency (RAl) in patients with septic shock is high and has a significant impact on survival. The purpose of this study was to determine whether a random cortisol concentration $<25 \mu \mathrm{g} / \mathrm{dL}$ was as good as a low dose $(1 \mu \mathrm{g})$ corticotrophin stimu- 
lation test in the diagnosis of RAl in patients with septic shock as assessed by the hemodynamic response to hydrocortisone.

METHODS: Patients were randomized to a single cortisol determination or to a low dose corticotrophin stimulation test. After blood collection to cortisol determinations, hydrocortisone (100 mg every $8 \mathrm{hrs}$ ) was administered for all patients in the first 36 hours. RAI was defined by a random cortisol concentration $<25$ $\mu \mathrm{g} / \mathrm{dL}$ or a $\Delta$ cortisol concentration $<9 \mu \mathrm{g} / \mathrm{dL}$ in the corticotrophin test.

RESULTS: Sixty patients (G1 = 30; G2 $=30$ ) were included in the analysis and were comparable regarding to demographic data, nosologies and disease severity. The time to norepinephrin withdrawal in group 1 patients with RAI diagnostic criteria was not different from the patients with cortisol $\geq 25 \mu \mathrm{g} / \mathrm{dL}$. In group 2 patients with $\Delta$ cortisol $<9 \mu \mathrm{g} / \mathrm{dL}$ had a shorter time of norepinephrin infusion (3 days) compared to patients with $\Delta$ cortisol $>9$ ( 6 days).

CONCLUSIONS: This study suggests that $1 \mu \mathrm{g}$ corticotrophin test is better than a random cortisol determination $<25 \mu \mathrm{g} / \mathrm{dL}$ to the diagnosis of relative adrenal insufficiency in septic shock patients.

Key Words: Diagnosis, relative adrenal insufficiency, septic shock.

\section{INTRODUÇÃO}

Vários estudos já mostraram que a incidência de insuficiência adrenal em pacientes com choque séptico é elevada e tem impacto significativo sobre a mortalidade $^{1,2}$. Annane e col. ${ }^{3}$ analisaram 299 pacientes com choque séptico e identificaram $76 \%$ dos pacientes como portadores de insuficiência adrenal relativa, ou seja, com resposta adrenal insuficiente para a intensidade do estresse. Nesse estudo a utilização de hidrocortisona e fludrocortisona reduziram a mortalidade em significativos $10 \%$.

Considera-se hoje como padrão ouro para a caracterização de insuficiência adrenal relativa no paciente com choque séptico, uma elevação do cortisol plasmático menor do que $9 \mu \mathrm{g} / \mathrm{dL}$ após administração de corticotropina $(250 \mu \mathrm{g})$, ou como vem sendo sugerido em estudos mais recentes, na dose de $1 \mu g^{4}$, quando comparado ao valor obtido antes da administração do hormônio.

A corticotropina deixou de ser comercializada no Brasil há vários anos, criando grandes dificuldades para a realização do teste no dia-a-dia das unidades de terapia intensiva (UTI). Marik e Zaloga ${ }^{5}$ ao analisarem a resposta ao teste da corticotropina com a utilização de $1 \mu \mathrm{g}$ ou $249 \mathrm{\mu g}$ em pacientes com choque séptico, observaram que a dosagem única do cortisol plasmático, utilizando-se para o diagnóstico um valor inferior a $25 \mu \mathrm{g} / \mathrm{dL}$, identificou insuficiência adrenal em $61 \%$ dos pacientes; enquanto que, com a utilização do teste da corticotropina com 1 $\mu \mathrm{g}$ do hormônio, $22 \%$ dos casos foram identificados. Quando o teste da corticotropina foi realizado com $249 \mu \mathrm{g}$ somente $8 \%$ dos casos foram caracterizados como insuficiência adrenal.

O objetivo deste estudo foi de comparar a eficiência da determinação única do cortisol plasmático, utilizandose como referência uma concentração plasmática < 25 $\mu \mathrm{g} / \mathrm{dL}$, com o teste da corticotropina com a utilização de 1 ㅆg do hormônio para a caracterização de insuficiência adrenal relativa em pacientes com choque séptico. A comparação foi feita avaliando-se a resposta hemodinâmica à administração de baixas doses de hidrocortisona. Avaliou-se secundariamente a mortalidade, tempo de permanência na UTI e a ocorrência de disfunções orgânicas após o diagnóstico do choque séptico.

\section{MÉTODO}

Após aprovação do protocolo do estudo pelo Comitê de Ética em Pesquisa do Hospital São Domingos, foi realizado um estudo prospectivo aleatório controlado, incluindo todos os pacientes acima de 18 anos de idade, não gestantes, que apresentaram quadro de choque séptico durante o período de internação em uma UTI geral de 20 leitos de um hospital de clínicas, e uma UTI de 11 leitos de um centro de trauma.

Foram excluídos do estudo além dos pacientes abaixo de 18 anos de idade e gestantes, os pacientes em tratamento com corticóide nos últimos 30 dias, aqueles que fizeram uso de etomidato nas 24 horas que precederam o momento da classificação, e os pacientes com diagnóstico prévio de doença do eixo hipotálamohipófise-adrenal.

O quadro de choque séptico foi caracterizado por hipotensão arterial (PA sistólica $<90 \mathrm{mmHg}$ ou PA média $<65 \mathrm{mmHg}$ ), que se manteve após uma hora de expansão volêmica (pelo menos $1.000 \mathrm{~mL}$ de solução fisiológica, substituto do plasma ou albumina a 5\%), mas que melhorou após uso de fármaco vasoativo, associado a: febre (temperatura $>$ de $38^{\circ} \mathrm{C}$ ) ou hipotermia (temperatura $<$ de $36^{\circ} \mathrm{C}$ ), leucocitose $\left(>12.000 / \mathrm{mm}^{3}\right)$ 
ou leucopenia $\left(<4.000 / \mathrm{mm}^{3}\right)$, infecção comprovada, ou seja, quadro clínico altamente sugestivo (pneumonia, abdômen agudo, obstrução urinária, abscesso, pus visível ou sugerido por exame de imagem), acrescido de confirmação microbiológica (cultura positiva, Gram ou histopatológico) ou, infecção fortemente suspeitada (quadro clínico altamente sugestivo), porém sem confirmação microbiológica (cultura negativa com antibioticoterapia já iniciada ou quando não foi colhido material para cultura).

Definido o quadro de choque séptico e após consentimento livre e esclarecido obtido de familiar próximo, os pacientes foram submetidos à aleatorização, através de tabela de números aleatórios, gerada por computador e envelopes lacrados para:

Grupo 1 - Realizada coleta de sangue para determinação da concentração plasmática de cortisol. Em seguida o paciente passava a receber $100 \mathrm{mg}$ de hidrocortisona, por via venosa a cada 8 horas. Após 36 horas da primeira dose de hidrocortisona, já de posse do resultado da dosagem do cortisol plasmático:

- Se o exame mostrava valor igual ou maior que $25 \mathrm{\mu g} /$ $\mathrm{dL}$ e o paciente continuava dependente de vasopressor, a administração de hidrocortisona era suspensa (Subgrupo 1a);

- Caso o cortisol estivesse abaixo de $25 \mu \mathrm{g} / \mathrm{dL}$, a hidrocortisona era mantida por 7 dias ou por 3 dias após a suspensão da infusão de noradrenalina, o que ocorresse primeiro. Em seguida a dose da hidrocortisona foi reduzida para $50 \mathrm{mg}$ a cada 8 horas, por 2 dias e a seguir suspensa (Subgrupo 1b);

- O paciente no qual a dosagem do cortisol foi igual ou maior que $25 \mu \mathrm{g} / \mathrm{dL}$, mas respondeu à administração de hidrocortisona, sendo possível suspender a infusão de noradrenalina durante as primeiras 36 horas de tratamento, teve a administração de hidrocortisona mantida por mais 3 dias, sendo em seguida a dose reduzida para $50 \mathrm{mg}$ a cada 8 horas por dois dias e em seguida suspensa (Subgrupo 1c).

Grupo 2 - Realizada coleta de sangue para determinação da concentração plasmática de cortisol, seguida de administração de $1 \mu \mathrm{g}$ de corticotropina, por via venosa (Synacthen; Novartis Pharma, Switzerland). Amostras de sangue para dosagem do cortisol plasmático foram coletadas 30 e 60 minutos após. Em seguida iniciava-se a administração de hidrocortisona (100 mg), por via venosa a cada 8 horas. Após 36 horas, com os resultados das dosagens de cortisol:

- Caso em alguma das dosagens pós-corticotropina o cortisol plasmático se elevasse acima de $9 \mu \mathrm{g} / \mathrm{dL}$ em relação ao basal, e o paciente continuasse dependente do vasopressor a hidrocortisona era suspensa (Subgrupo 2a);

- Caso o cortisol pós-corticotropina fosse igual ou menor que $9 \mu \mathrm{g} / \mathrm{dL}$, a hidrocortisona era mantida por sete dias, ou por três dias após a suspensão da infusão de noradrenalina, o que ocorresse primeiro, sendo em seguida a dose reduzida para $50 \mathrm{mg}$ a cada 8 horas por mais dois dias, após os quais o fármaco era suspenso (Subgrupo 2b);

- O paciente que, em resposta ao teste da corticotropina, elevou o cortisol plasmático acima de $9 \mu \mathrm{g} / \mathrm{dL}$, que respondeu a administração de hidrocortisona, sendo possível suspender a infusão de vasopressor durante as primeiras 36 horas de tratamento, a administração de hidrocortisona foi mantida por mais 3 dias sendo em seguida reduzida para $50 \mathrm{mg}$ a cada 8 horas por mais dois dias e a seguir suspensa (Subgrupo 2c).

O cortisol plasmático foi determinado por método de quimiluminescência. Todas as outras intervenções terapêuticas destinadas ao tratamento da sepse e choque séptico, incluindo antibioticoterapia, fármacos inotrópicos e vasopressores, monitorização hemodinâmica, terapia nutricional precoce, seguiram a mesma rotina nos dois serviços, que são atendidos pela mesma equipe médica e de enfermagem.

Os pacientes tiveram a gravidade definida pelo escore APACHE III e disfunções orgânicas que foram avaliadas na admissão à UTI e quando da definição do quadro de choque séptico, se este não tivesse sido o motivo da internação na UTI.

O escore APACHE III foi determinado conforme descrito originalmente por Knaus e col. ${ }^{6}$. Para a caracterização das disfunções orgânicas utilizaram-se os seguintes critérios: pulmonar (necessidade de ventilação mecânica por pelo menos 24 horas), renal (necessidade de diálise - excluindo-se os pacientes renais crônicos já em tratamento dialítico), hepática (bilirrubinemia $>3$ mg\% + TGO e TGP > 2 vezes o valor normal).

Para definir a disfunção orgânica após inclusão em um dos grupos do estudo, o critério de definição deveria estar ausente até o momento da aleatorização.

\section{Análise Estatística}

Os dados são apresentados como média \pm desvio padrão, proporção ou mediana com intervalos interquartis. O teste Exato de Fisher foi usado para avaliar variáveis categóricas e o teste $t$ de Student para as variáveis contínuas paramétricas. $\mathrm{Na}$ análise dos 6 
subgrupos resultantes das determinações do cortisol plasmático utilizou-se a Análise da Variância (ANOVA). O tamanho da amostra calculado foi de 60 casos (30 em cada grupo) para análise unicaudal. Os testes estatísticos foram considerados significativos para $p<$ 0,05. A versão 11.0 do SPSS (SPSS Inc., Chicago II, USA) foi utilizada para as análises.

\section{RESULTADOS}

No período de junho de 2006 a maio de 2007, 63 pacientes internados nas duas UTI do estudo tiveram confirmado o diagnóstico de choque séptico. Três pacientes foram excluídos do estudo, dois deles devido a não obtenção do consentimento livre e esclarecido e um devido ao uso de etomidato durante procedimento cirúrgico pouco antes da internação na UTI com choque séptico. Trinta pacientes foram aleatorizados para cada um dos grupos. A tabela 1 mostra que os dois grupos são comparáveis com relação à idade, sexo, escore APACHE III e local da infecção.

Com relação ao tempo de uso de noradrenalina no grupo 1 não se observou diferença entre o subgrupo 1a (pacientes sem critério diagnóstico de insuficiência adrenal) e o subgrupo 1b (aqueles com cortisol plasmático $<25 \mu \mathrm{g} / \mathrm{dL}$ ). Entretanto, quando se analisaram os subgrupos a e b do grupo 2 , os pacientes com critério diagnóstico de insuficiência adrenal (não responsivos ao teste da corticotropina) tiveram expressiva redução do tempo de uso de noradrenalina quando comparados àqueles sem o critério (3 dias versus 6 dias), embora a Análise de Variância não tenha revelado diferença estatisticamente significativa (Tabela 2). A análise da mortalidade e do tempo de permanência na UTI não permitiu conclusões, a não ser a de que fossem estudados grupos de pacientes realmente muito graves, com mortalidade bastante elevada. Nos dois subgrupos de pacientes identificados como portadores de insuficiência adrenal relativa ( 1 b e 2b), a incidência de disfunções orgânicas foi mais elevada que nos subgrupos sem critérios de insuficiência adrenal. Novamente as diferenças não alcançaram significância estatística. Um dado que chamou a atenção foi a existência de três pacientes no subgrupo 1c, ou seja, pacientes nos quais a dosagem única do cortisol mostrou valor acima de $25 \mu \mathrm{g} / \mathrm{dL}$ e que ao final de 36 horas de uso de corticosteróide já não utilizavam fármaco vasopressor (seriam falsos negativos à dosagem única de cortisol),

Tabela 1 - Dados Demográficos

\begin{tabular}{|c|c|c|c|}
\hline Variáveis & $\begin{array}{l}\text { Grupo } 1 \text { - Dosagem Única } \\
\qquad(n=30)\end{array}$ & $\begin{array}{l}\text { Grupo } 2 \text { - Teste de Corticotropina } \\
\qquad(\mathrm{n}=30)\end{array}$ & Valor de $p$ \\
\hline Idade (anos) ${ }^{\star}$ & $62,5 \pm 3,3$ & $56,7 \pm 3,8$ & 0,25 \\
\hline $\operatorname{Sexo}(M / F)$ & $17 / 13$ & $11 / 19$ & 0,19 \\
\hline APACHE III* & $81,7 \pm 4,4$ & $84,5 \pm 5,1$ & 0,68 \\
\hline \multicolumn{4}{|l|}{ Local da infecção } \\
\hline Abdômen & 12 & 9 & \\
\hline Pulmão & 9 & 9 & \\
\hline Partes moles & 5 & 4 & \\
\hline Urina & 2 & 7 & \\
\hline Não identificado & 1 & 1 & \\
\hline
\end{tabular}

*Valores expressos em média \pm DP

Tabela 2 - Resultados

\begin{tabular}{|c|c|c|c|c|c|c|c|c|}
\hline & \multicolumn{4}{|c|}{ Grupo 1} & \multicolumn{4}{|c|}{ Grupo 2} \\
\hline & \multicolumn{4}{|c|}{ Subgrupos } & \multicolumn{4}{|c|}{ Subgrupos } \\
\hline & $\begin{array}{c}1 \mathrm{a} \\
\mathrm{n}=12\end{array}$ & $\begin{array}{c}1 \mathrm{~b} \\
\mathrm{n}=15\end{array}$ & $\begin{array}{c}1 \mathrm{c} \\
\mathrm{n}=3\end{array}$ & $\mathrm{p}$ & $\begin{array}{c}2 a \\
n=10\end{array}$ & $\begin{array}{c}2 b \\
n=19\end{array}$ & $\begin{array}{c}2 c \\
n=1\end{array}$ & $\mathrm{p}$ \\
\hline Mortalidade n (\%) & $9(75)$ & $9(60)$ & $1(33)$ & 0,20 & $5(50)$ & $12(63)$ & 0 & 0,3 \\
\hline $\begin{array}{l}\text { Disfunção orgânica pós-cho- } \\
\text { que séptico (n) }\end{array}$ & 1 & 3 & 1 & 0,5 & 2 & 4 & - & 0,6 \\
\hline
\end{tabular}

$I Q=$ Intervalo interquartil. 
enquanto no grupo 2 apenas um paciente apresentou o teste da corticotropina falso negativo, ou seja, apresentou um $\Delta$ do cortisol $>9 \mu \mathrm{g} / \mathrm{dL}$, mas respondeu a administração de hidrocortisona.

\section{DISCUSSÃO}

Embora não seja possível estabelecer conclusões definitivas com base nos resultados deste estudo, foram identificadas evidências de que o teste da corticotropina com $1 \mu \mathrm{g}$ do hormônio pode ser mais eficiente para estabelecer o diagnóstico de insuficiência adrenal relativa no paciente com choque séptico, quando comparado à dosagem única do cortisol, pelo menos quando se utiliza uma concentração plasmática $<25$ $\mu \mathrm{g} / \mathrm{dL}$ como critério na dosagem única. Essas evidências seriam representadas pela redução expressiva do tempo de infusão de noradrenalina no subgrupo $2 b$ (não responsivos a corticotropina) comparado ao subgrupo 2a (responsivo à corticotropina), enquanto que os subgrupos $1 \mathrm{a}$ (cortisol plasmático $\geq 25 \mu \mathrm{g} / \mathrm{dL}$ ) e $1 \mathrm{~b}$ (cortisol plasmático $<25 \mu \mathrm{g} / \mathrm{dL}$ ) praticamente não diferiram com relação ao tempo de uso de vasopressor. Além disso, no grupo submetido a dosagem única de cortisol, três pacientes (10\%) apresentaram resposta falso negativa, ou seja, cortisol plasmático $>25 \mu \mathrm{g} / \mathrm{dL}$ mas que, ao final de 36 horas de uso de hidrocortisona já não utilizavam fármaco vasopressor, enquanto no grupo submetido a teste da corticotropina somente um paciente (3\%) apresentou resposta falso negativa, ou seja, tinha um $\Delta$ do cortisol $>9 \mu \mathrm{g} / \mathrm{dL}$, mas respondeu a administração de hidrocortisona.

A deficiência de cortisol, o principal glicocorticóide produzido pelo córtex adrenal, está associada a aumento da mortalidade em pacientes com choque séptico ${ }^{2,7}$. $O$ funcionamento normal da glândula adrenal é essencial para a sobrevivência. A administração prolongada de fármacos que suprimem a função adrenal está associada a aumento da mortalidade de pacientes graves ${ }^{8}$. Na década de 1980, três estudos prospectivos, aleatórios, duplamente encobertos utilizaram corticosteróide em pacientes com choque séptico ${ }^{9-11}$. Nenhum desses estudos mostrou beneficio com relação à mortalidade e somente um deles identificou redução do tempo de duração do choque no grupo de pacientes que recebeu corticosteróide. Todos esses estudos utilizaram doses elevadas de corticosteróides por curto espaço de tempo.

Nos anos 1990 o interesse pelos corticosteróides como tratamento adjuvante no choque séptico foi renovado, agora utilizando doses menores de hidrocortisona por período de tempo mais prolongado. Dois estudos com casuísticas limitadas (aproximadamente 40 pacientes em cada estudo) compararam corticosteróide com placebo em pacientes com choque séptico e observaram redução na duração do choque nos pacientes que receberam corticosteróide ${ }^{12,13}$.

Embora esteja estabelecida a faixa de normalidade do cortisol plasmático em indivíduos normais, não há concordância sobre qual seria o nível desejável de cortisol para o paciente grave. A maioria dos autores considera que é muito difícil estabelecer um valor único de cortisol plasmático que possa separar pacientes graves com ou sem deficiência relativa do hormônio. Os valores variam de 10 até $34 \mu \mathrm{g} / \mathrm{dL}^{14-16}$. Em estudo envolvendo 299 pacientes com choque séptico, Annane e col. ${ }^{3}$ concluíram que pacientes submetidos ao teste da corticotropina com $250 \mu \mathrm{g}$ do hormônio e que não apresentavam elevação do cortisol plasmático acima de 9 $\mu \mathrm{g} / \mathrm{dL}$, tinham a sobrevida expressivamente melhorada quando eram tratados com a associação de hidrocortisona e fludrocotisona. Mostrou mais, que os pacientes com resposta normal ao teste da corticotropina, não apresentavam benefícios do uso de corticóide.

O uso de dose elevada de corticotropina para realização do teste resulta em níveis de ACTH muitas vezes mais elevados no plasma que aqueles vistos no paciente submetido a estresse intenso. Essa dose pode resultar em um superestímulo da glândula adrenal produzindo uma resposta falsa negativa ao teste. A utilização de $1 \mu \mathrm{g}$ de corticotropina resulta em nível plasmático de ACTH muito semelhante àquele encontrado no paciente com choque séptico. Tordjman e col. ${ }^{17}$ avaliaram o desempenho do teste da corticotropina utilizando $250 \mu \mathrm{g} \mathrm{e} 1 \mu \mathrm{g}$ para diagnosticar falha do eixo hipotálamo-hipófise-adrenal. O teste foi realizado em indivíduos sadios, pacientes com doença hipofisária e função normal do eixo e pacientes com doença hipofisária e disfunção do eixo. A sensibilidade do teste com $1 \mu \mathrm{g}$, para identificar disfunção do eixo foi de $94,7 \%$ versus $6,2 \%$ do teste com $250 \mu \mathrm{g}$. Sireaux e col. ${ }^{4} \mathrm{com}-$ pararam o teste da corticotropina com $250 \mu \mathrm{g}$ e com 1 $\mu \mathrm{g}$ em um grupo de pacientes com choque séptico. $\mathrm{O}$ teste com $1 \mu \mathrm{g}$ foi expressivamente mais eficiente para diagnosticar insuficiência adrenal relativa nesses pacientes. Marik e Zaloga ${ }^{5}$, analisando 59 pacientes com choque séptico, submetidos a teste com 1 e $249 \mu \mathrm{g}$ de corticotropina e comparado a pacientes com cortisol plasmático basal menor do que $25 \mu \mathrm{g} / \mathrm{dL}$, mostraram que o cortisol abaixo de 25, diagnosticou insuficiência adrenal relativa em percentual expressivamente maior 
que os testes com 1 e $249 \mu$ de corticotropina.

O CORTICUS, estudo multicêntrico recentemente publicado $^{18}$, que envolveu 52 serviços na Europa e Oriente Médio e analisou um total de 500 pacientes, ao comparar pacientes com choque séptico submetidos à corticoterapia com pacientes que receberam placebo, mostrou melhora da sobrevida no grupo de pacientes que receberam hidrocortisona, mas não confirmou os dados de Annane e col. com relação à utilidade do teste da corticotropina, para identificar pacientes que se beneficiam daqueles que não se beneficiam do uso de corticosteróide. Apesar disso o estudo mostrou uma incidência de infecção nosocomial mais elevada nos pacientes tratados com corticosteróide além de níveis glicêmicos mais elevados. Ainda assim, baseado nos resultados do estudo CORTICUS, a versão mais recente do Surviving Sepsis Campaign ${ }^{19}$ recomenda a utilização de corticosteróide em todo paciente com choque séptico que não responde ao vasopressor. Essa recomendação aparentemente não leva em conta nem as complicações infecciosas observadas no estudo CORTICUS, e nem outros estudos que relacionam o uso de corticosteróides a aumento de morbidade e mortalidade em pacientes graves de uma forma geral ${ }^{20,21}$.

\section{CONCLUSÃO}

O presente estudo sugere maior eficiência do teste da corticotropina com $1 \mu \mathrm{g}$, quando comparado a dosagem única de cortisol para detecção de insuficiência adrenal relativa em pacientes com choque séptico, embora a significância estatística não tenha sido alcançada.

Concorda-se com Annane, que durante recente conferência (Canada Critical Care Forum - outubro 2007), afirmou que se deve procurar estabelecer um método confiável para diagnosticar essa síndrome, com o objetivo de beneficiar, com o uso de doses suprafisiológicas de corticosteróide, somente os pacientes com insuficiência adrenal relativa, evitando-se o uso nos pacientes que não só não se beneficiarão da intervenção, como estarão sujeitos a aumento do risco de infecção nosocomial pelo uso desnecessário de esteróides.

\section{REFERÊNCIAS}

01. Rothwell PM, Udwadia ZF, Lawler PG - Cortisol response to corticotropin and survival in septic shock. Lancet, 1991;337:582-583.

02. Annane D, Sébille V, Troché $G$ et al - A 3-level prognostic classification in septic shock based on cortisol levels and cortisol response to corticotrophin. JAMA, 2000;283:1038-1045.

03. Annane D, Sébille V, Charpentier $C$ et al - Effect of treatment with low doses of hydrocortisone and fludrocortisone on mortality in patients with septic shock. JAMA, 2002;288:862-871.

04. Siraux V, De Backer D, Yalavatti G et al - Relative adrenal insufficiency in patients with septic shock: comparison of low-dose and conventional corticotropin tests. Crit Care Med, 2005;33: 2479-2486.

05. Marik PE, Zaloga GP - Adrenal insufficiency during septic shock. Crit Care Med, 2003;31:141-145.

06. Knaus WA, Wagner DP, Draper EA et al - The APACHE III prognostic system. Risk prediction of hospital mortality for critically ill hospitalized adults. Chest, 1991;100:1619-1636.

07. Zaloga GP, Marik P - Hypothalamic-pituitary-adrenal insufficiency. Crit Care Clin, 2001;17:25-41.

08. Ledingham IM, Watt I - Influence of sedation on mortality in critically ill multiple trauma patients. Lancet, 1983;1:(8336):1270.

09. Sprung CL, Caralis PV, Marcial EH et al - The effects of high-dose corticosteroids in patients with septic shock. A prospective, controlled study. N Engl J Med, 1984;311:1137-1143.

10. Bone RC, Fisher CJ Jr, Clemmer TP et al - A controlled clinical trial of high-dose methylpredinisolone in the treatment of severe sepsis and septic shock. N Engl J Med, 1987;317:653-658.

11. Effect of high-dose glucocorticoid therapy on mortality in patients with clinical signs of systemic sepsis. The Veterans Administration Systemic Sepsis Cooperative Study Group. N Engl J Med, 1987;317:659-665.

12. Bollaert PE, Charpentier C, Levy B et al - Reversal of late septic shock with supraphysiologic doses of hydrocortisone. Crit Care Med, 1998;26:645-650.

13. Briegel J, Forst $\mathrm{H}$, Haller $\mathrm{M}$ - Stress doses of hydrocortisone reverse hyperdynamic septic shock: a prospective, randomized, double-blind, single-center study. Crit Care Med, 1999;27:723-732.

14. Beishuizen A, Thijs LG - Relative adrenal failure in intensive care: an identifiable problem requiring treatment? Best Pract Res Clin Endocrinol Metab, 2001;15:513-531.

15. Knowlton AL - Adrenal insufficiency in the intensive care setting. J Intensive Care Med, 1989;4: 35-41.

16. Kidess Al, Caplan $\mathrm{RH}$, Reynertson $\mathrm{RH}$ et al - Transient corticotropin deficiency in critical illness. Mayo Clin Proc, 1993;68:435-441.

17. Tordjman $\mathrm{K}$, Jaffe $\mathrm{A}$, Trostanetsky $\mathrm{Y}$ et al - Low-dose (1 microgram) adenocorticotrophin (ACTH) stimulation as a screening test for impaired hypothalamo-pituitary-adrenal axis function: sensitivity, specificity and accuracy in comparison with the high-dose (250 microgram) test. Clin Endocrinol, 2000;52:633-640.

18. Sprung CL, Annane D, Keh D, et al - Hydrocortisone therapy for patients with septic shock. N Engl J Med, 2008;358:111-124.

19. Dellinger RP, Levy M, Carlet JM, et al. Surviving sepsis campaign: international guidelines for management of severe sepsis and septic shock: 2008. Crit Care Med, 2008;36:296-327.

20. Roberts I, Yates D, Sandercock P et al - Effect of intravenous corticosteroids on death within 14 days in 10008 adults with clinically significant head injury (MRC CRASH trial): randomised placebo-controlled trial. Lancet, 2004;364:1321-1328.

21. Rady MY, Johnson DJ, Patel B et al - Corticosteroids influence the mortality and morbidity of acute critical illness. Crit Care, 2006;10:R101. 